\title{
Modal conversion of a phase-locked extended-cavity diode laser array into a single lobe
}

\author{
David Pabœuf, Florian Emaury, Sébastien de Rossi, Arnaud Jérome, Michel Lamare, \\ Raymond Mercier, Gaëlle Lucas-Leclin and Patrick Georges \\ Laboratoire Charles Fabry de l'Institut d'Optique, CNRS, Univ Paris-Sud, Campus Polytechnique - \\ RD128 - 91127 Palaiseau Cedex, France
}

\begin{abstract}
Phase-locked operation of an array of ten diode lasers is demonstrated in an extended-cavity using the Talbot selfimaging effect. An output power up to $1.7 \mathrm{~W}$ has been obtained. The extracavity coherent conversion of the multilobed array supermode into a Gaussian mode is investigated theoretically based on a binary phase grating. The best configuration results in a conversion efficiency of $83 \%$. Experimentally, the conversion efficiency reaches $50 \%$ and is limited by the imperfect coherence of the laser array. We conclude that the conversion setup provides an actual measurement of the power in the selected array supermode.
\end{abstract}

\section{INTRODUCTION}

The constant and growing demand for efficient and powerful laser sources leads to explore new architectures aiming at increasing the output power of lasers diodes. The most promising approach is to use multiple moderate-power, highbrightness, lasers and then recombine them in one single diffraction-limited beam. Different beam combining techniques have been thus investigated in the literature, from which passive external-cavity configurations are usually preferred for their simplicity. Though spectral beam combining has resulted to date in the highest brightness with diode laser arrays [1], this technique has the major drawback of its inherently wide spectrum. On the other hand passive coherent combining configurations, which consist in inducing a constant phase relation-ship between the sources, offer an interesting alternative, and various extended-cavity setups have been demonstrated $[2,3,4]$. Nevertheless, the far-field profile resulting from the coherent combination of several lasers consist in multiple interference peaks, because of the non-uniform near field emissive aperture. This is particularly critical with diode laser bars, which have low fill factors for proper thermal management. Then the total coherent power is distributed within numerous peaks, and the on-axis intensity of the laser beam remains low. Efficient conversion of the multilobed beam into a Gaussian profile is then necessary to further improve the beam quality.

Several techniques aiming at converting the emission from a low fill factor coherent laser array into a single lobe have already been proposed. One can classify them into aperture-filling techniques and superposition techniques. The aim of former is to generate a uniform near field emissive area, in phase and intensity, by filling the gaps between the lasers [5]. The other solution consists in the superposition of the different beams from each laser by use of a diffractive optical element (DOE). Indeed, DOE are usually used to split a beam into several beams propagating at different angles corresponding to the diffraction orders of the grating. A proper design of the phase profile of the DOE provides a fine control of the amplitude and phase of each diffraction order. Thanks to the reciprocity of light propagation, this optical device can also be used to combine several coherent laser beams on top of one another [3,6].

In this paper, we describe the passive coherent combining of 10 diode lasers in an external cavity using the Talbot selfimaging effect. Furthermore, we realize the extracavity coherent superposition of the 10 emitters with a DOE. We demonstrate efficient conversion of the multilobed array supermode into a Gaussian-like mode, up to a power of $365 \mathrm{~mW}$. We explain the lower conversion efficiency, as compared to theory, by the low coherence of the laser array emission.

\section{DESCRIPTION OF THE LASER SOURCE}

The laser source is an array of $M=10$ index-guided tapered lasers emitting around $975 \mathrm{~nm}$ with a pitch $\mathrm{p}=100 \mu \mathrm{m}$. The active layer consists of a strained GaInAs quantum well embedded in a large optical cavity. The lateral structure of the emitters is a tapered ridge which overall length is $2.5 \mathrm{~mm}$ [7]. The rear facet is high-reflection coated ( $\mathrm{R}>90 \%)$. Thanks to the antireflection coating $\left(\mathrm{R}<10^{-3}\right)$ on the front facet of the laser array, no laser emission but only amplified 
spontaneous emission (ASE) is observed without external cavity. The beam from the laser array is collimated in the fast axis direction with a high NA acylindric lens.

The phase locking between the emitters is induced with the help of a passive external cavity, using the Talbot selfimaging effect $[4,8]$; the extended-cavity simply consists of a volume Bragg grating (VBG) which acts as the external cavity output coupler, positioned at a quarter of the Talbot distance $Z_{T}=2 p^{2} / \lambda \cong 20 \mathrm{~mm}$ from the laser array front facet to ensure the highest discrimination between the in-phase and the out-of-phase supermodes (see Figure 1). The VBG reflectivity is $40 \%$ at $976 \mathrm{~nm}$, with a spectral bandwidth $\Delta \lambda=0.3 \mathrm{~nm}$; its angular acceptance is $\Delta \theta=3^{\circ}$ (full width at $\left.1 / \mathrm{e}^{2}\right)$, which is larger than the divergence of the laser array in both directions after fast axis collimation. The extendedcavity laser array threshold is $0.9 \mathrm{~A}$, and the output power reaches $1.7 \mathrm{~W}$ at the operating current of $3.9 \mathrm{~A}$ [9]. Depending on the angle of the VBG, the extended-cavity may operate either in the out-of-phase or the in-phase array supermode.

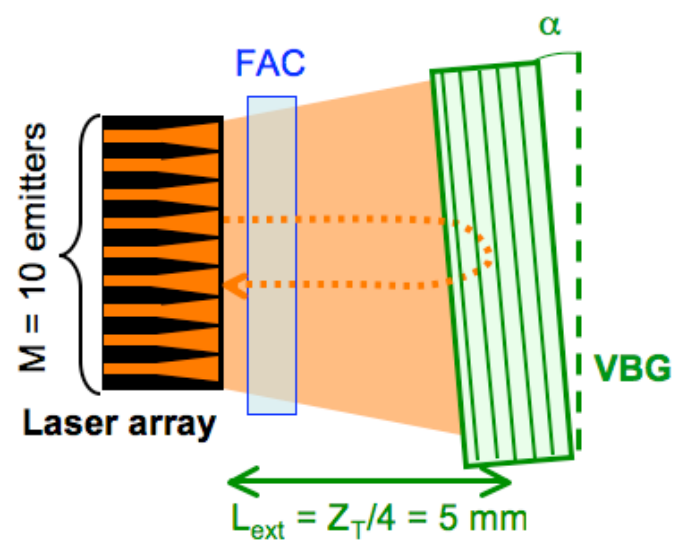

Figure 1 : Experimental setup of the Talbot extended-cavity laser array with a volume Bragg grating; FAC : fast axis collimation lens; VBG : volume Bragg grating.

The far-field profile in the slow-axis is measured with a CCD camera at the Fourier plane of a cylindrical collimating lens; it exhibits narrow interference peaks, which is a clear evidence of the phase locking of the laser array (see Figure 2). The 150-mrad wide envelope of the far-field profile corresponds to the incoherent far-field emission of the individual lasers; thus the number of peaks is related to the low fill factor of the array $(2 \mathrm{w} / \mathrm{p}=30 \%)$ and the slightly multimode emission of the tapered ridges. Consequently no more than $15 \%$ of the total power is included in the central lobe. The full width at half maximum of each peak is close to the diffraction angular limit of the array at low currents (1 mrad). At high output powers $(\mathrm{P}>1.2 \mathrm{~W})$, the incoherent contribution of the free-running array operation induces a parasitic pedestal in the far-field distribution; moreover the interference peaks are slightly broadened to $2.5 \mathrm{mrad}$. In addition the envelope of the far field presents some distortion, which is attributed to a degradation of the beam quality of the emitters.

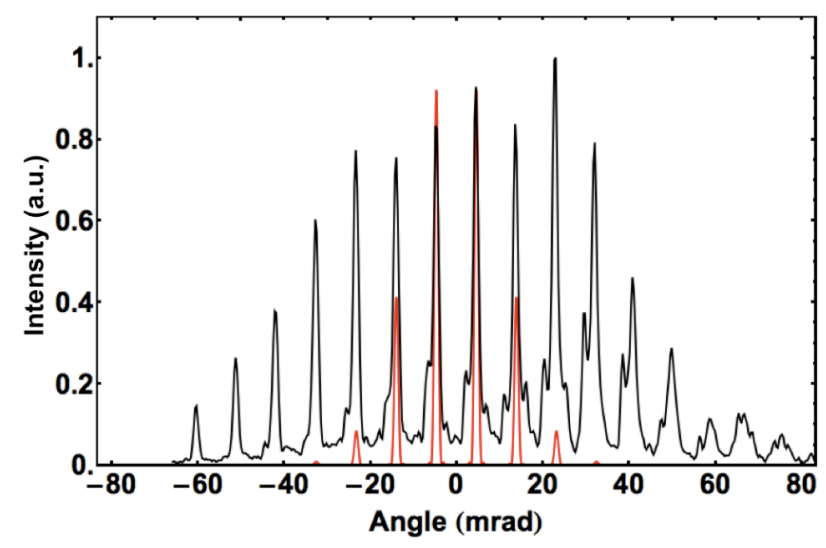

Figure 2 : Experimental far-field profile of the extended-cavity laser array in the slow axis at I=2 A; in red, theoretical farfield profile. 


\section{DESIGN OF THE COHERENT CONVERSION SETUP}

Our coherent combination setup is described on Figure 3. It is based on a phase grating $\Phi_{2}$, placed in the Fourier plane of the collimating lens $\mathrm{L}_{3}$. The grating aims at superimposing the beams from the $\mathrm{M}$ emitters on one; behind the focusing lens $\mathrm{L}_{4}$, the near-field image $\mathrm{NF}^{\prime}$ consists in one main spot with most of the optical power. The conversion will be efficient if only the phases, amplitudes and angles of incidence of the $M$ beams match the diffraction pattern of the grating. Thus it might be necessary to have an additional phase mask $\left(\Phi_{1}\right)$ onto the intermediate near-field plane NF to adapt the phases of each beam. It is actually a coherent conversion of the laser array supermode into a Gaussian mode, which is possible thanks to the high coherence of the array emission.
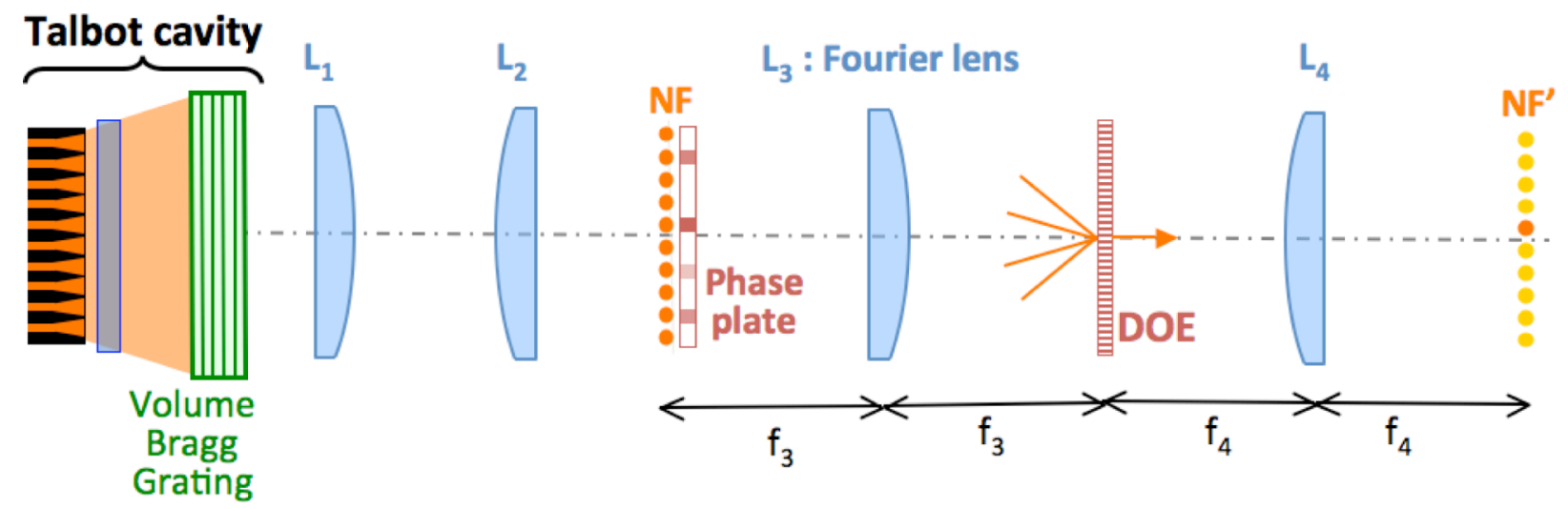

Figure 3 : Experimental setup for the coherent conversion of the array supermode; NF, NF' : near-field images; DOE : diffractive optical element; $L_{i}(i=1$ to 4$)$ : cylindrical lens with focal length $f_{i}$.

The M supermodes of the extended-cavity laser array may be calculated with a coupled-mode analysis, based on the calculation of the eigenmodes of the laser equation [10]. They are actually very similar to the ones deduced in the simplest case assuming only adjacent coupling between emitters [11] :

$$
E_{n}(x)=\sum_{k=1}^{M} \sin \left(\frac{n}{M+1} k \pi+\frac{n \pi}{2}\right) \times \exp \frac{\left(x+\left(\frac{M+1}{2}-k\right) p\right)^{2}}{w^{2}}, 1 \leq n \leq M
$$

where $\mathrm{M}$ is the number of emitters, $\mathrm{w}$ is their waist radius, $\mathrm{p}$ is the array pitch and the origin, $\mathrm{x}=0$, is defined at the center of the laser array. $n=1$ represents the in-phase mode whereas $n=M$ is the out-of-phase mode. The near-field and far-field profiles of the latter are drawn on Figure 4, with $M=10, w=15 \mu \mathrm{m}$ and $\mathrm{p}=100 \mu \mathrm{m}$ which correspond to our experimental parameters. Both in-phase and out-of-phase modes have the same near-field intensity profile, which has a sinusoidal envelop $\cos ^{2}(\pi \mathrm{x} /(\mathrm{M}+1) \mathrm{p})$. 

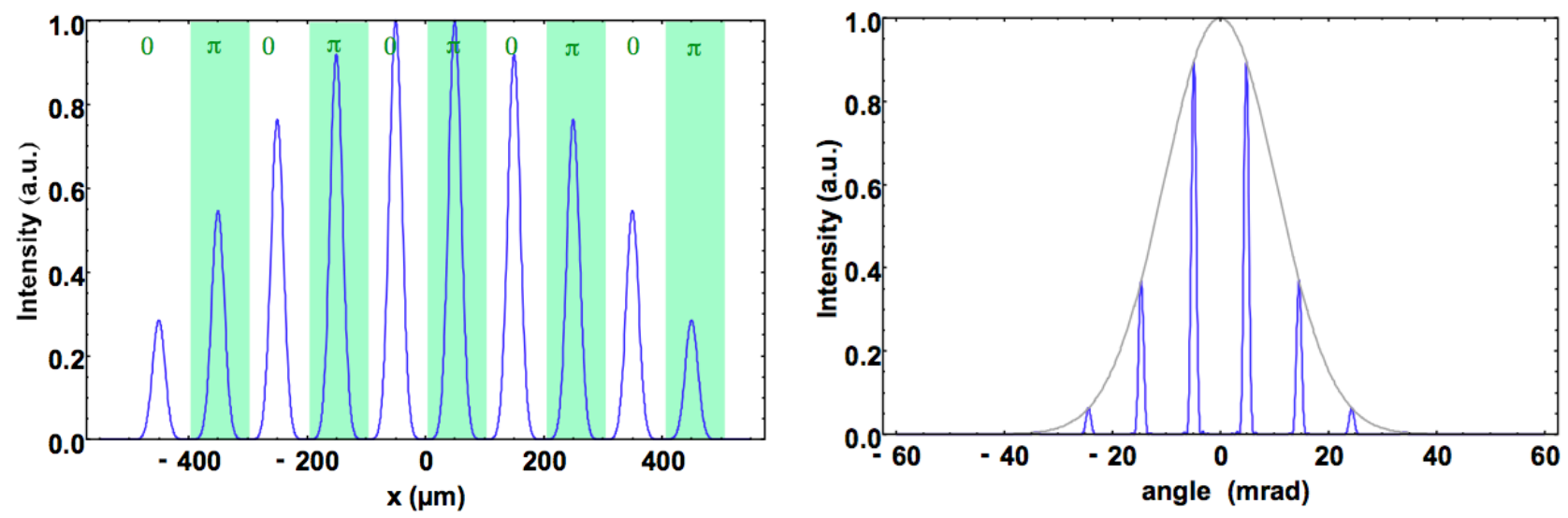

Figure 4: Theoretical near-field (left) and far-field (right) intensity profiles of the out-of-phase mode, with $\mathrm{M}=10, \mathrm{w}=15 \mu \mathrm{m}$, $\mathrm{p}=100 \mu \mathrm{m}, \lambda=976 \mathrm{~nm}$

As the phase grating, we will consider a simple $(0, \pi)$ binary grating - or diffractive optical element (DOE) [12]; its period $a$ is related to the focal length of the Fourier lens $L_{3}$ and the array pitch $p$, following the relationship $a=f_{3} \times \lambda / p$; the motive of the grating is characterized by its $\mathrm{N}$ phase-shift positions $\left\{\mathrm{x}_{\mathrm{S}}, \mathrm{s}=1 \ldots \mathrm{N}\right\}$. The amplitudes of the diffraction orders of such a grating are given by [13]

$$
\begin{gathered}
\tau_{0}=1-2 \sum_{\mathrm{s}=1}^{\mathrm{N}}(-1)^{\mathrm{s}} \frac{\mathrm{x}_{\mathrm{s}}}{\mathrm{a}} \\
\tau_{\mathrm{n}}=\frac{1}{\mathrm{i} \pi \mathrm{n}} \sum_{\mathrm{s}=1}^{\mathrm{N}}(-1)^{\mathrm{s}} \mathrm{e}^{-2 \mathrm{i} \pi \frac{\mathrm{x}_{\mathrm{s}}}{\mathrm{a}}}, \quad \mathrm{n} \in \mathrm{Z}^{*}
\end{gathered}
$$

An algorithm has been implemented to determine the phase profile $\left\{x_{s}\right\}$ of the best grating, which diffraction pattern $\left\{\tau_{\mathrm{n}}\right\}$ matches the theoretical near field intensity profile of either the in-phase or out-of-phase mode. The phase state required in the near field plane is given by the relative phases of the diffraction orders of the DOE. The efficiency of the solution is evaluated by the combination efficiency $\eta$ defined as the ratio between the power $P_{\text {comb }}$ combined into the main spot in the imaged near field plane NF' of Figure 3 and the total optical power $P_{\text {total }}$ in that very plane $\eta=P_{\text {comb }} / P_{\text {tot }}$. Our best solution results in a theoretical combining efficiency $\eta_{0}=83 \%$; it consists of one $\pi$ phase step $\left\{x_{1} / a=0.41\right.$, $\left.\mathrm{x}_{2} / \mathrm{a}=0.59\right\}$ and requires the following phase state in the NF plane : $\{\pi, 0, \pi, 0,0,0, \pi, 0, \pi, 0\}$, which neither corresponds to the in-phase mode nor the out-of-phase mode but is actually close to the latter with the $5^{\text {th }}$ emitter phase-shifted by $\pi$. A simple phase mask, with one $\pi$ phase step, has to be used in the NF plane to adapt the phase state of the near field. In Figure 5, we compare the theoretical near field profiles obtained in NF' with and without the DOE, after propagation of a beam with the previously determined phase state. It is obvious that the DOE allows combining most of the optical power into a single spot. The remaining power is distributed into additional peaks corresponding to replicas of the diffraction orders of the grating.

We have also numerically studied the sensitivity of the conversion setup to manufacturing defects of the phase filters $\Phi_{1}$ and $\Phi_{2}$. In fact the combination efficiency remains above $70 \%$ as long as the phase height is between $[0.7 \times \pi ; 1.3 \times \pi]$ and the motive width $\left(\mathrm{x}_{2}-\mathrm{x}_{1}\right) / \mathrm{a}$ does not change of more than $30 \%$, which are precisions much looser than the typical manufacturing tolerances of such components. Similarly the combination efficiency is not very sensitive to a random phase shift of one beam as long as it remains within $\pm 0.2 \times \pi$ of its nominal value. 


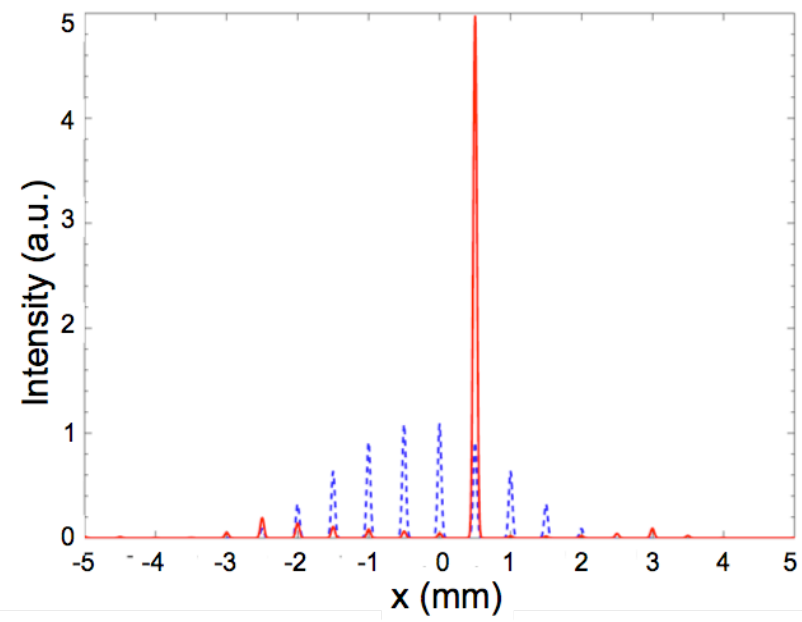

Figure 5: Theoretical near-field profiles in the NF' plane of the conversion setup, without (dashed blue line) and with (red solide line) the DOE.

\section{EXPERIMENTAL VALIDATION}

The DOE has been manufactured by Holoeye Photonics according to our design; its period a is $488 \mu \mathrm{m}$ and the motive width is $88 \mu \mathrm{m}$. From its diffraction pattern we deduce a diffraction efficiency of $\eta_{0}=83 \%$ in accordance with the theoretical value. The phase mask has been realized in our laboratory by ion etching of a BK7 plate. It consists of a phase step of $0.99 \times \pi$ at $\lambda=976 \mathrm{~nm}$, and a width of $80 \mu \mathrm{m}$. Neither the phase plate nor the DOE are antireflection coated; we evaluate the transmission of the optical system to $\mathrm{T}=85 \%$. The Talbot external cavity laser array is aligned in order to operate in the out-of-phase mode. The near field of the array is imaged with a $\times 1$ afocal lens $\left(\mathrm{L}_{1}, \mathrm{~L}_{2}\right)$ on the phase plate $\Phi_{1}$, which shifts of $\pi$ the phase of the $5^{\text {th }}$ emitter only.

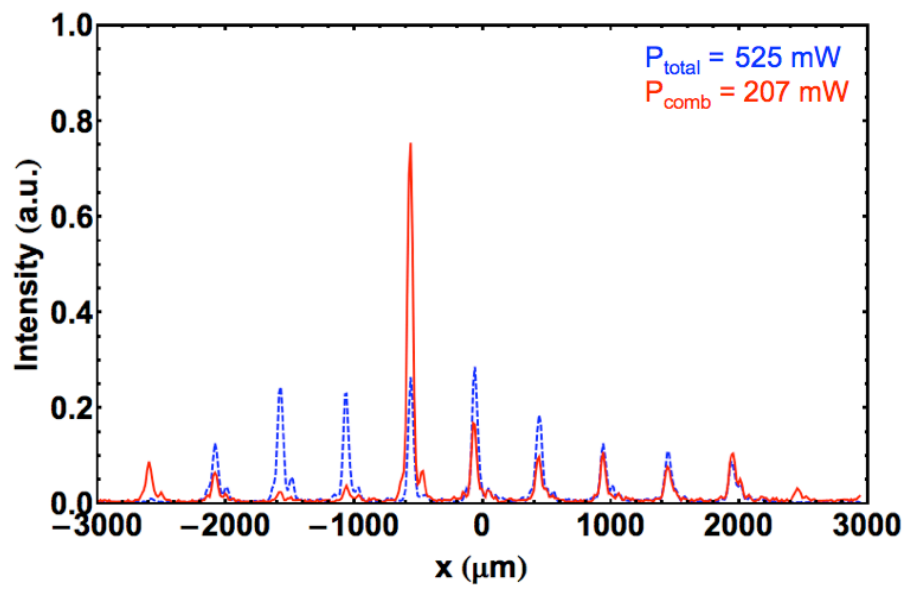

Figure 6: Experimental slow-axis near-field profile in the plane NF' at I = 2 A, without (blue dashed line) and with (red solid line) the DOE.

Figure 6 shows the near-field profiles imaged in NF' without the DOE, at the operating current $\mathrm{I}=2 \mathrm{~A}$; the total power is distributed among the $M=10$ emitters, and roughly follows the theoretical cosine profile. With the DOE inserted in the conversion setup, most of the optical power is within one major peak, as expected. The beam quality factor of the combined beam has been measured to $\mathrm{M}^{2}=1.7$ in the slow axis, close to the value of the individual emitters [7]. The ratio $\eta$ of the power which is actually collected into that peak has been measured as a function of the operating current of the extended-cavity array (see Figure 7): close to threshold, the combining efficiency reaches $\eta=50 \%$, and it remains above $37 \%$ at the maximum operating current. The maximum power in the combined spot reaches $310 \mathrm{~mW}$ at $\mathrm{I}=3 \mathrm{~A}$, 
without correction from the $\mathrm{T}=85 \%$ transmission of the whole setup $-\mathrm{P}_{\text {comb }}=365 \mathrm{~mW}$ with correction. This is significantly larger than previous results of coherent combining into one peak from an array of diode lasers [3]. The decline of $\eta$ with the operating current indicates a fall in the coherence of the laser array as the output power is increased. Indeed since only the out-of-phase supermode can be efficiently converted to a Gaussian-like mode, the overall combining efficiency is proportional to the ratio $\mathrm{C}$ of power which is actually in the out-of-phase supermode at the output of the extended-cavity array. With a DOE efficiency $\eta_{0}=83 \%$, we can deduce that C is between $60 \%$ and $40 \%$. This could have been predicted from the experimental far-field profile of the extended-cavity laser array in the outof-phase mode shown in Figure $2:$ it is wider than expected due to a slightly multimode operation of the tapered ridge emitters; in addition, an incoherent pedestal is noticeable, and the contribution of other supermodes is evidenced from the supplementary peaks close to the main ones. Our conversion setup provides thus a measurement of the coherence of the phase-locked laser array, by quantifying the power contained in the chosen supermode.

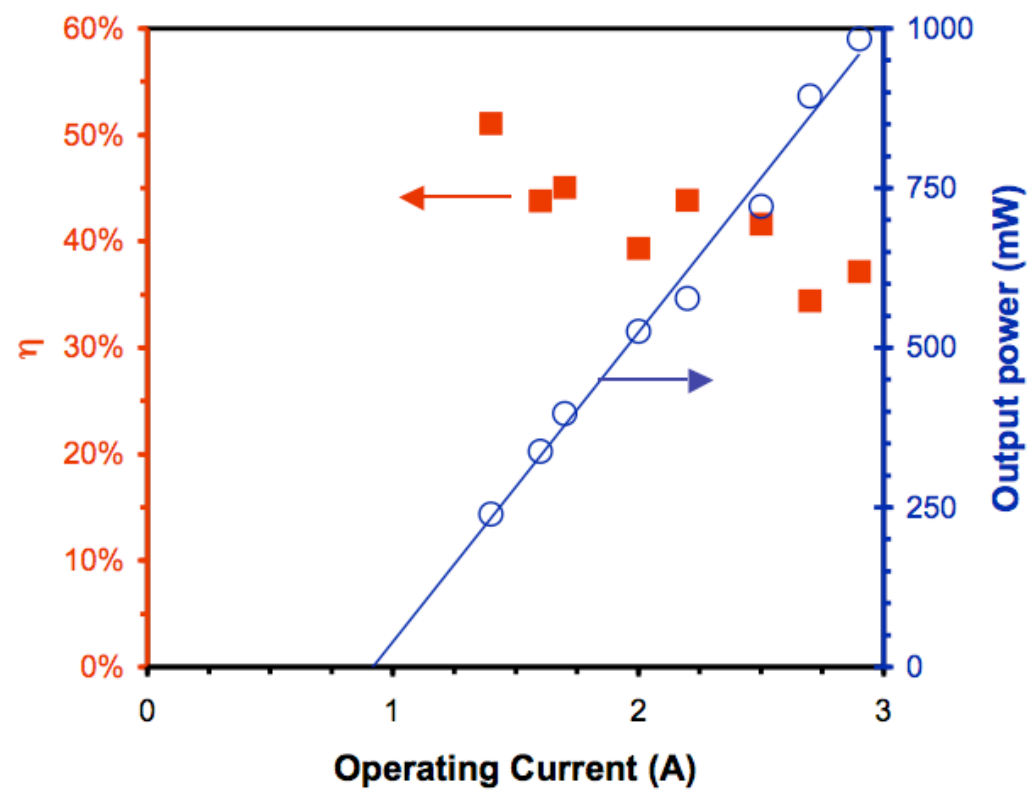

Figure 7: Evolution of experimental conversion efficiency $\eta$ within the out-of-phase mode (red full square, left) and the output power of the extended-cavity laser array (blue circle, right) with the operating current.

\section{CONCLUSION}

We have described a simple extended-cavity laser array configuration which aims at inducing a strong phase-locking of 10 diode lasers; it is based on the self-imaging Talbot effect, which produces a strong coupling between all the emitters to force a coherent operation of the whole array. In addition we use a volume Bragg grating to lock the laser line. The intrinsic multilobed profile - both in the near field and in the far field - of the phase-locked array emission has been coherently converted to a Gaussian-like mode in a conversion setup which makes use of a binary phase grating to superpose the 10 emitters onto one. A specific DOE has been designed, and a theoretical conversion efficiency of $83 \%$ has been calculated. Experimentally, the conversion efficiency is limited by the imperfect coherence of the laser array; this is attributed to the competition of the phase-locked operation of the laser array in the out-of-phase supermode against incoherent self-running and other array supermodes. We evaluate that up to $60 \%$ of the total power at the output of the extended-cavity laser is actually in the - selected - out-of-phase mode. The proposed conversion setup provides thus an actual measurement of the coherence of the laser array.

\section{ACKNOWLEDGMENTS}

This work was supported within the European project WWW.BRIGHTER.EU under Grant No. FP6-IST-035266. D. Pabœuf thanks the French Ministry of Defense (DGA) for funding his Ph.D. 


\section{REFERENCES}

1. R. K. Huang, B. Chann, L. J. Missaggia, J. P. Donnelly, C. T. Harris, G. W. Turner, A. K. Goyal, T. Y. Fan and A. Sanchez-Rubio, "High-brightness wavelength beam combined semiconductor laser diode arrays", IEEE Photonics Technology Letters, 19(2-4), 209-211 (2007).

2. C. J. Chang Hasnain, J. Berger, D. R. Scifres, W. Streifer, J. R. Whinnery, and A. Dienes, "High-power with high-efficiency in a narrow single-lobed beam from a diode-laser array in an external cavity", Applied Physics Letters 50 (21), 1465-1467 (1987).

3. J. R. Leger, G. J. Swanson, and W. B. Veldkamp, "Coherent laser addition using binary phase gratings," Applied Optics 26, 4391-4399 (1987).

4. R. Waarts, D. Mehuys, D. Nam, D. Welch, W. Streifer, and D. Scifres, "High-power, CW, diffraction-limited, GaAlAs laser diode-array in an external Talbot cavity", Applied Physics Letters 58 (23), 2586-2588 (1991).

5. M. Khajavikhan, A. Hoyer-Leitzel and J. R. Leger, "Efficient conversion of light from sparse laser arrays into single-lobed far field using phase structures", Optics Letters, 33(20), 2377-2379 (2008).

6. G. Hergenhan, B. Lucke, and U. Brauch, "Coherent coupling of vertical-cavity surface-emitting laser arrays and efficient beam combining by diffractive optical elements: concept and experimental verification," Applied Optics 42, 1667-1680 (2003).

7. M. Krakowski, S. C. Auzanneau, F. Berlie, M. Calligaro, Y. Robert, O. Parillaud and M. Lecomte, " 1 W high brightness index guided tapered laser at $980 \mathrm{~nm}$ using Al-free active region materials", Electronics Letters, 39(15), 1122-1123 (2003).

8. R.K. Huang, B. Chann, L.J. Missagia, S.J. Augst, R.B. Swint, J.P. Donnelly, A. Sanchez-Rubio, and G.W. Turner, "High-Power Coherent Beam Combination of Semiconductor Laser Arrays", presented at the Conference on Lasers and ElectroOptics (2008).

9. D. Paboeuf, G. Lucas-Leclin, P. Georges, N. Michel, M. Krakowski, J. Lim, S. Sujecki, and E. Larkins, "Narrow-line coherently combined tapered laser diodes in a Talbot external cavity with a volume Bragg grating," Applied Physics Letters 93, 211102 (2008).

10. D. Mehuys, W. Streifer, R. G. Waarts and D. F. Welch, "Modal-analysis of linear Talbot-cavity semiconductorlasers", Optics Letters, 16(11), 823-825 (1991)

11. J. K. Butler, D. E. Ackley, and D. Botez, "Coupled-mode analysis of phase-locked injection-laser arrays," Applied Physics Letters 44, 293-295 (1984).

12. H. Dammann and K. Görtler, "High-efficiency in-line multiple imaging by means of multiple phase holograms," Optics Communications 3, 312-315 (1971).

13. S. Martellucci and A. N. Chester, Diffractive optics and optical microsystems, The language of science, Plenum Press, New York, p. 422 (1997) 